\title{
Preeclampsia in pregnancy and later use of antihypertensive drugs
}

\author{
Anders Engeland ${ }^{1,2,6} \cdot$ Tone Bjørge ${ }^{2,3} \cdot$ Kari Klungsøyr $^{2,4} \cdot$ Rolv Skjærven $^{2,4}$ • \\ Svetlana Skurtveit $^{1,5} \cdot$ Kari Furu $^{1}$
}

Received: 26 October 2014/Accepted: 10 March 2015/Published online: 18 March 2015

(c) The Author(s) 2015. This article is published with open access at Springerlink.com

\begin{abstract}
We explored the association between preeclampsia and later use of antihypertensive drugs in a population-based study with data from the Medical Birth Registry of Norway and the Norwegian Prescription Database. The study cohort consisted of 980,000 women having 2.1 million pregnancies during 1967-2012. Hazard ratios (HRs) with $95 \%$ confidence intervals $(95 \% \mathrm{CI})$ were estimated in multivariate time-dependent Cox proportional hazards regression models. Overall, the HR of later use of antihypertensive drugs was 2.0 (95\% CI 2.0-2.0) in women with one preeclamptic pregnancy compared to women without preeclamptic pregnancies. The HR increased by increasing number of preeclamptic pregnancies, both term and preterm pregnancies. In women with two or more preeclamptic pregnancies, the HR was 2.8 (2.7-3.0). The overall HR after 40 years of follow-up for women with one preeclamptic pregnancy was 1.3
\end{abstract}

Anders Engeland

anders.engeland@igs.uib.no

1 Division of Epidemiology, Department of Pharmacoepidemiology, Norwegian Institute of Public Health, Oslo, Norway

2 Department of Global Public Health and Primary Care, University of Bergen, Bergen, Norway

3 Cancer Registry of Norway, Oslo, Norway

4 Medical Birth Registry of Norway, Division of Epidemiology, Norwegian Institute of Public Health, Bergen, Norway

5 Norwegian Centre for Addictive Research, University of Oslo, Oslo, Norway

6 Division of Epidemiology, Department of Pharmacoepidemiology, Norwegian Institute of Public Health, Kalfarveien 31, 5020 Bergen, Norway
(1.2-1.4) and for two or more preeclamptic pregnancies the HR was $1.6(1.1-2.1)$. The first 5 years after the first birth, the HR of being dispensed antihypertensive drugs was higher in preterm [8.4 (7.7-9.1)] than term preeclamptic pregnancies [4.3(4.0-4.6)]. However, after 10 years, this difference was no longer present. The HR of later use of antihypertensive drugs increased with the number of preeclamptic pregnancies, and in the first 10 years the HR was higher after a preterm than a term preeclamptic pregnancy. Although the HR decreased with time since first birth, the risk was still elevated after 40 years.

Keywords Preeclampsia - Antihypertensive drugs . Prescriptions · Population-based registries

$\begin{array}{ll}\text { Abbreviations } \\ \text { ATC } & \text { Anatomical Therapeutic Chemical } \\ \text { CI } & \text { Confidence interval } \\ \text { HR } & \text { Hazard ratio } \\ \text { ICD } & \text { International Classification of Diseases } \\ \text { MBRN } & \text { Medical Birth Registry of Norway } \\ \text { NorPD } & \text { Norwegian Prescription Database } \\ \text { PE } & \text { Preeclampsia }\end{array}$

Introduction

About $3 \%$ of Norwegian pregnancies are complicated by preeclampsia (PE) [1], a condition characterized by maternal hypertension and proteinuria after the 20th week of pregnancy. The disease is a leading cause of maternal as well as foetal morbidity and mortality. Preeclampsia has in particular been shown to be associated with later maternal 
hypertension [2-4], ischemic heart disease, cerebrovascular disease, type 2 diabetes mellitus, chronic renal failure [5] and cardiovascular mortality [6]. Preeclampsia is one of the most deadly hypertensive complications of pregnancy and increases the risk of later cardiovascular disease two to four times [7].

Preeclampsia is associated with severe endothelial dysfunction, and these endothelial changes might increase the risk of later cardiovascular disease [8]. Williams [9] discussed how PE can predict later maternal health, and emphasized the need for women with a history of PE to make changes in lifestyle. Bellamy et al. [4], found it likely that women having recurrent $\mathrm{PE}$ had a pathological phenotype that puts them at risk of hypertension. After a review of 13 studies, they reported a relative risk of 3.7 of later maternal hypertension associated with a history of PE. The included studies were cohort studies (including case-cohort and nested case-control studies) with a mean follow-up time of 14 years. A population-based cohort study of 3400 women [10], it was found that women with a history of hypertensive disorders in pregnancy had higher blood pressure and body-mass index than those without. In a more recent review [11], it was reported a relative risk of 3.1 of maternal hypertension after PE.

The aim of our study was to explore possible relations between PE associated with preterm or term delivery and later maternal use of antihypertensive drugs in a large population-based cohort study with long follow-up using national registries in Norway. We also wanted to explore whether a possible relation changed with number of pregnancies with $\mathrm{PE}$, number of births and time since first childbirth.

\section{Materials and methods}

\section{Data sources}

The Norwegian Central Population Registry (NCPR) contains demographic information on all residents in Norway, including date of birth and date of emigration or death [12].

The Norwegian Prescription Database (NorPD) [13] contains information on all prescribed drugs, dispensed at pharmacies to individual patients treated in ambulatory care, both public and private, from January 1st, 2004, and covers the entire Norwegian population (approximately 5.0 million individuals in 2012). Data on use in institutionalized patients in nursing homes and hospitals are also collected, but these figures are only registered at an institutional level. Therefore, drugs dispensed at institutions are not included in our study. For each prescription, age of the patient, demographic information, dispensing date, and detailed drug information are registered. Classification of drugs is based on the Anatomical Therapeutic
Chemical (ATC) classification system [14]. In this study, we included the following drugs (hereafter denoted 'antihypertensive drugs'): Antithrombotic agents (ATC-code: $\mathrm{B} 01)$, antihypertensives (C02), diuretics (C03), beta blocking agents (C07), calcium channels blockers (C08) and agents acting on the renin-angiotensin system (C09). In Norway, medications for chronic diseases are reimbursed, and only reimbursed medications were included here.

The Medical Birth Registry of Norway (MBRN) is a population-based registry containing information on all births in Norway since 1967 (more than 2.7 million births), except most induced abortions. MBRN is based on compulsory notification of every birth, from 16 completed weeks of gestation onwards, and includes identification of the parents by their personal identification numbers, demographic information on the parents, maternal health prior to and during pregnancy, length of pregnancy, delivery as well as information on the infant, including birth defects and other adverse pregnancy outcomes [15]. Until late 1998, PE was notified to MBRN either as "preeclampsia" or the combination of "hypertension" and "proteinuria". Since late 1998, MBRN defines PE as an increase in blood pressure to at least 140 systolic and/or $90 \mathrm{mmHg}$ diastolic (or an increase in diastolic blood pressure $\geq 15 \mathrm{mmHg}$ from the level measured before 20th gestational week), combined with proteinuria (at least 0.3 gram per $24 \mathrm{~h}$ or $\geq 1+$ on dipstick).

From late 1998, a new notification form based on check boxes was introduced and PE was notified by the alternatives "Preeclampsia, mild", "Preeclampsia, severe" or "Preeclampsia, before 34 weeks", and with separate check boxes for HELLP (hemolysis, elevated liver enzymes and low platelet count) syndrome and eclampsia. The new notification form also included information on maternal smoking in association with the pregnancies, as well as ultrasound based estimation of gestational age.

In the present study, we denote pregnancies with $\mathrm{PE}$ $(2.8 \%)$, eclampsia $(0.03 \%)$ and HELLP syndrome $(0.05 \%)$ as preeclamptic $(2.8 \%)$. Further, PE was categorized as preterm or term PE based on whether the delivery occurred before 37 or at 37 completed weeks or more, respectively [6]. Gestational age was based on the last menstrual period (LMP) until 1998, and thereafter primarily on ultrasound estimation, but on LMP if ultrasound estimation was missing.

\section{Study subjects}

Data in NorPD, MBRN and NCPR were linked using the unique 11-digit personal identification number, assigned to all individuals living in Norway (Fig. 1).

In our study, mothers of all singleton pregnancies ending in a birth during 1967-2012 with birth weight of $500 \mathrm{~g}$ (the 


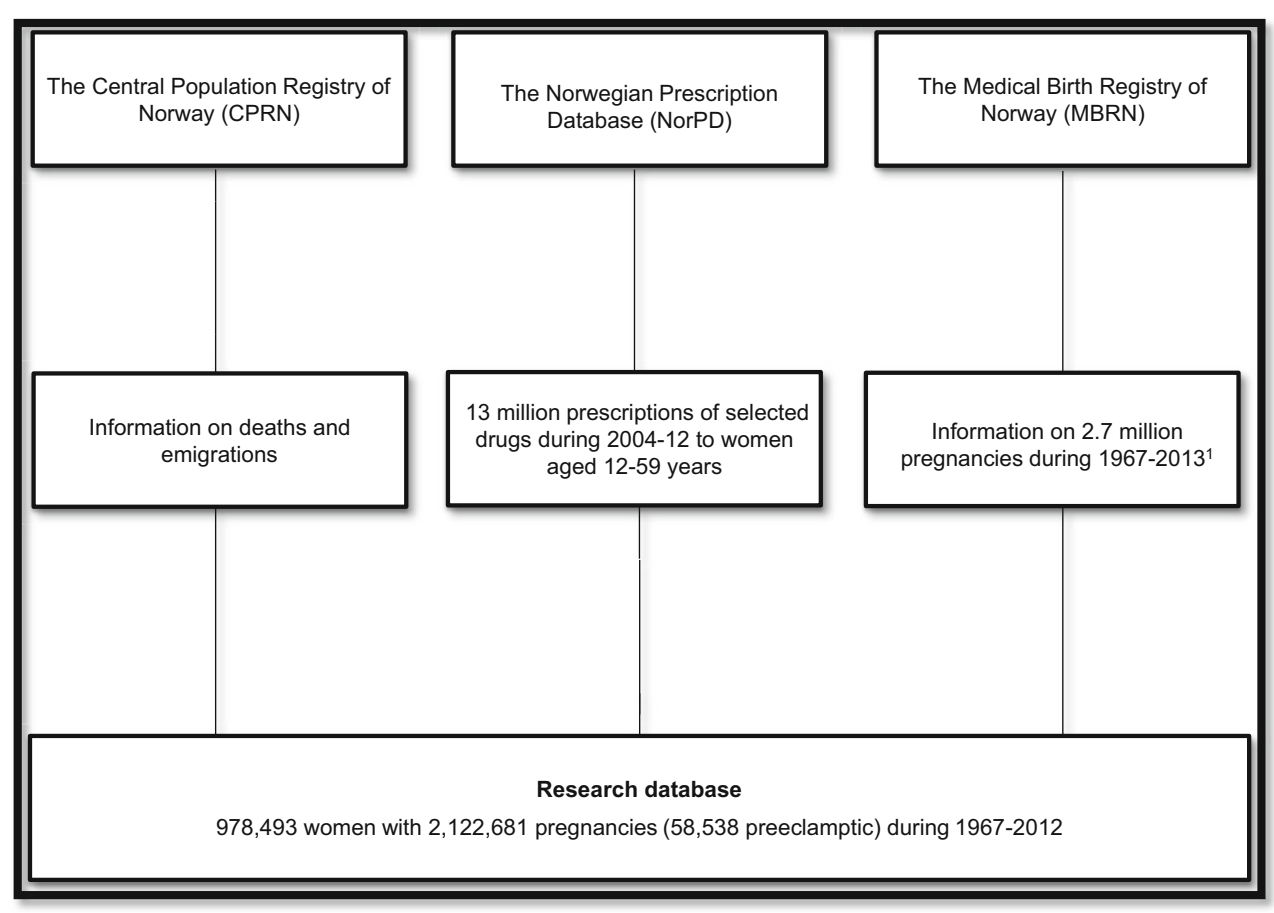

Fig. 1 Data sources. ${ }^{1}$ Mothers of all singleton pregnancies ending in a birth during 1967-2012, were included in our study. However, births in 2013 were also used to have information on when new pregnancies in 2012 started, when constructing the observation time for the analyses

mean weight of foetuses at about 22 completed weeks of gestation [16]) or more, were included, provided that the mothers were still living in Norway later than July 1, 2004, and that their first birth was registered in MBRN. Births in 2013 were also used to obtain information on when new pregnancies in 2012 started for constructing the observation time until censoring.

Pregnancies where maternal heart diseases or maternal chronic hypertension were diagnosed prior to the specific pregnancy or antihypertensive drugs were dispensed to the mother prior to the specific pregnancy, were excluded ( $8 \%$ of the pregnancies). Dispensed antihypertensive drugs during pregnancy were excluded as well.

In Norway, medicines for chronic diseases are usually dispensed for a maximum of 3 months at the time. To ensure that none of the women in our study population had hypertension before pregnancy we chose to exclude those having been dispensed antihypertensive drugs between January 1 and June 30, 2004. A total of 978,493 mothers remained (Fig. 1).

Information on previous preeclamptic pregnancies was obtained from births in MBRN during 1967-2012. All mothers were followed, with respect to the dispensing of antihypertensive drugs in the NorPD, from their first registered birth or July 1, 2004, whichever occurred latest (i.e. the data were left censored), until dispensed antihypertensive drugs, emigration, death or December 31, 2012 (Fig. 2). Time during pregnancies was excluded from the observation time. In all analyses, information from all previous births was used for each woman.

\section{Methods}

Hazard ratios (HRs) of being dispensed antihypertensive drugs were estimated using multivariate time-dependent Cox proportional hazards regression models. Time since first birth was used as time variable.

In the analyses, we adjusted for a number of potential confounders. We included previous maternal number of births, year of childbirth (1967-1976, 1977-1986, 1987-1996, 1997-2006 and 2007-2012) and number of preeclamptic pregnancies (none, one and two or more), in addition to maternal age and maternal age at first birth ( $<20$ years, $20-24, \ldots, 35-39$ and $\geq 40$ years). The impact of adjustment on the estimates was modest. We also made stratified/adjusted analyses including variables only available for parts of the study period (e.g. maternal smoking).

Since diabetes has been shown to be associated with the use of antihypertensive drugs, analyses with adjustment for maternal diabetes prior to pregnancy were performed.

From 1999, information on maternal smoking was available, and was used to stratify pregnancies on maternal daily smoking (yes/no).

Unfortunately, information on maternal weight and height was scarce and only available in a few years at the 


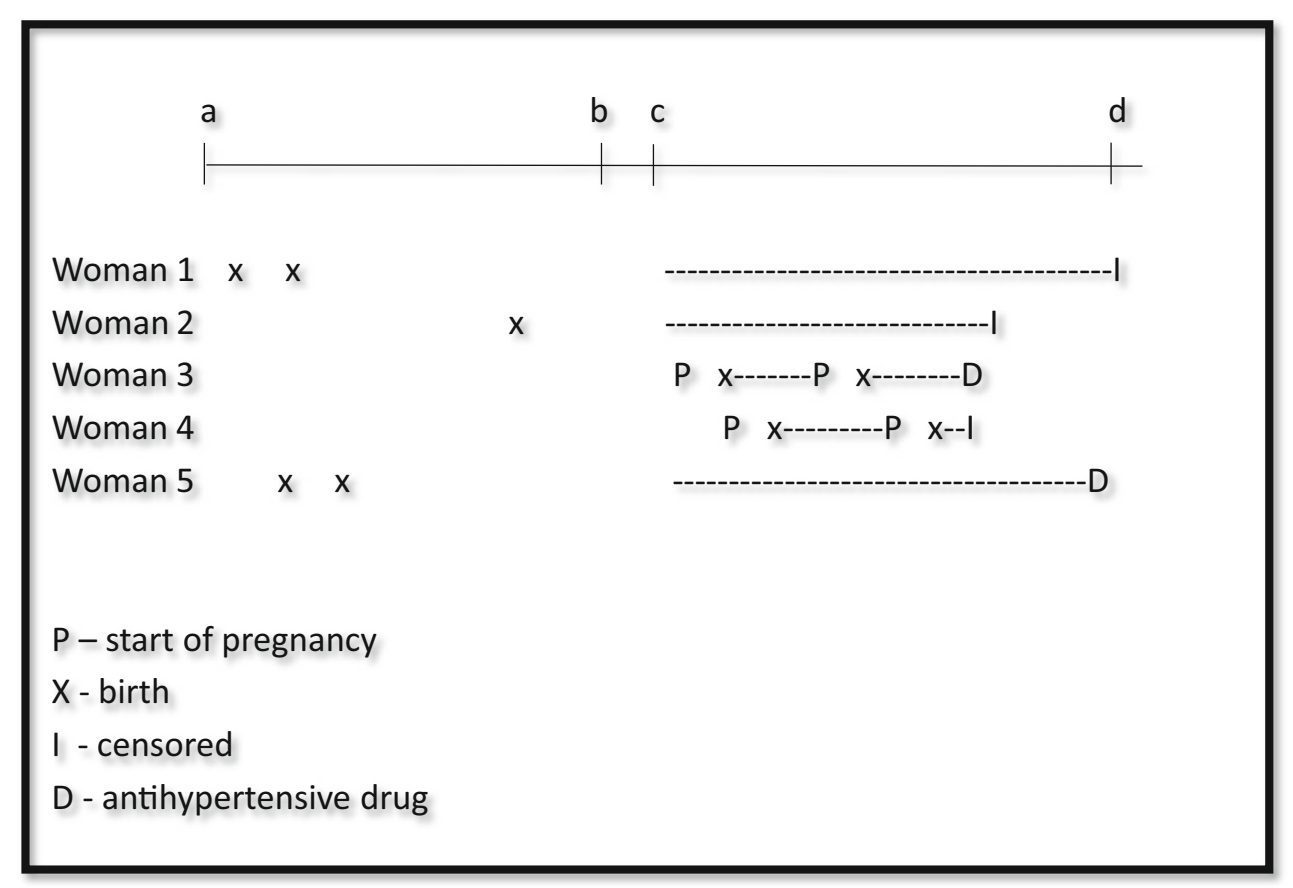

Fig. 2 Observation time (dashed line) in the Norwegian Prescription Database (NorPD) for women included in the study according to pregnancies during 1967-2012. Time-dependent variables were used to update information on the women at each birth. $a$ January 1, 1967,

end of the study period. Hence, the impact of maternal weight and height could not be addressed.

The main analysis was performed both with and without antithrombotic drugs (ATC-code: B01) included in the group of antihypertensive drugs.

Data handling and analyses were performed using IBM Statistics 20 and Stata/SE 12.1.

\section{Results}

This study included information on 2,122,681 births for 978,493 women. A total of 58,538 pregnancies were preeclamptic. Altogether 52,691 women had one or more preeclamptic pregnancies, $75 \%$ of these women had PE in their first pregnancy. The women in the study were followed in the NorPD for an average of 6.7 years (range 0-8.5 years), constituting 6.6 million person-years. Due to the start of follow-up of women in the NorPD in July 1st, 2004, or later (since NorPD was established in 2004), the mean start of follow-up in the NorPD was 14 years after the first birth of the women (range 0-38 years), and the total follow-up was up to 46 years after the first birth. Altogether 155,978 women were dispensed antihypertensive drugs during follow-up in NorPD (Table 1). $95 \%$ of the follow-up time was in women without any preeclamptic pregnancies (Table 2). start of the Medical Birth Registry of Norway. $b$ January 1, 2004, start of the Norwegian Prescription Database. $c$ July 1, 2004: Earliest start of follow-up. $d$ December 31, 2012, end of follow-up

The HR of being dispensed antihypertensive drugs increased with increasing number of preeclamptic pregnancies (Table 3). The HR was 2.0 (95\% CI 2.0-2.0) in women with one preeclamptic pregnancy compared to those without. In women with two or more preeclamptic pregnancies the HR was 2.8 (2.7-3.0). Excluding antithrombotic drugs (ATC-code: B01) from the group of antihypertensive drugs gave similar results (data not shown).

The HR was higher after preterm than term preeclamptic pregnancies (Table 4). The HR was $2.2(2.1-2.3)$ in women with one pregnancy with preterm preeclampsy compared to those without preeclamptic pregnancies. When only births after 1998 were included (after introducing the new notification form in MBRN), the corresponding HR was 3.3 (3.1-3.5). When including only the time from the first birth to the next pregnancy or a maximum of 5 years, the HR after preterm PE was 8,4 (7.7-9,1) and after term PE the HR was 4.3 (4.0-4.6).

The HR was highest in the first 5 years after the first birth and decreased gradually thereafter (Table 5). However, the women still had an increased HR 40 years after the first birth. After 40-44 years of follow-up, the overall HR of being dispensed antihypertensive drugs associated with one preeclamptic pregnancy was $1.3(1.2-1.4)$, and for two or more preeclamptic pregnancies the HR was 1.6 (1.1-2.1). In the first 5 years after the first birth, the HR of 
Table 1 Characteristics of the women and person-time of follow-up in the Norwegian Prescription Database (NorPD)

\begin{tabular}{|c|c|c|c|c|}
\hline & $\begin{array}{l}\text { Number of } \\
\text { women }\end{array}$ & $\begin{array}{l}\text { Number of women who were dispensed } \\
\text { antihypertensive drugs during } \\
\text { follow-up in the NorPD }\end{array}$ & Person-years & $\begin{array}{l}\text { Rate of dispensed } \\
\text { antihypertensive drugs } \\
\text { per } 1000 \text { person-years }\end{array}$ \\
\hline \multicolumn{5}{|c|}{ Year of birth (mother) } \\
\hline$<1950$ & 116,628 & 42,617 & 787,399 & 54.1 \\
\hline $1950-1959$ & 224,187 & 56,234 & $1,649,742$ & 34.1 \\
\hline 1960-1969 & 262,100 & 36,635 & $2,042,521$ & 17.9 \\
\hline 1970-1979 & 243,403 & 16,752 & $1,611,609$ & 10.4 \\
\hline $1980-$ & 132,175 & 3740 & 472,941 & 7.9 \\
\hline \multicolumn{5}{|c|}{ Year of childbirth ${ }^{\mathrm{a}}$} \\
\hline $1967-1976$ & 97,447 & 35,770 & 656,264 & 54.5 \\
\hline $1977-1986$ & 167,829 & 46,711 & $1,211,310$ & 38.6 \\
\hline 1987-1996 & 228,088 & 40,670 & $1,756,335$ & 23.2 \\
\hline 1997-2006 & 340,147 & 29,171 & $2,565,075$ & 11.4 \\
\hline 2007-2012 & 144,982 & 3656 & 375,229 & 9.7 \\
\hline \multicolumn{5}{|l|}{$A g e^{\mathrm{a}}$} \\
\hline$<20$ & 30,045 & 4004 & 186,354 & 21.5 \\
\hline $20-24$ & 179,681 & 28,234 & $1,140,733$ & 24.8 \\
\hline $25-29$ & 347,020 & 54,295 & $2,309,765$ & 23.5 \\
\hline $30-34$ & 293,559 & 45,887 & $2,034,923$ & 22.5 \\
\hline $35-39$ & 110,686 & 19,857 & 773,239 & 25.7 \\
\hline $40-44$ & 16,931 & 3567 & 115,593 & 30.9 \\
\hline $45+$ & 571 & 134 & 3606 & 37.2 \\
\hline Total & 978,493 & 155,978 & $6,564,212$ & 23.8 \\
\hline
\end{tabular}

a At first pregnancy registered in the Medical Birth Registry of Norway

being dispensed antihypertensive drugs was much higher in preterm PE $[\mathrm{HR}=8.3$ (95\% CI 7.6-9.1) and 13 (8.2-21) in one and two or more pregnancies with preterm PE, respectively] than in term PE [4.2 (3.9-4.5) and 8.1 (6.0-11), in one and two or more pregnancies with term PE, respectively]. However, after the first 10 years, there was no difference between term and preterm preeclamptic pregnancies.

\section{Discussion}

This population-based study included pregnancies registered in the MBRN during 1967-2012, with information on approximately 980,000 women with almost 59,000 preeclamptic pregnancies. An increased risk of later maternal use of antihypertensive drugs in women having had $\mathrm{PE}$ was observed. The increased risk decreased with time since first pregnancy, but the risk was still elevated 40 years later.

We used nationwide registries, MBRN and NorPD, based on compulsory notifications of all births in Norway and all prescribed drugs dispensed to individuals from all Norwegian pharmacies. A number of papers on the occurrence of PE have been published based on MBRN data $[6,17,18]$, and the diagnosis of PE for the time period 1967-2005 was validated in 2013 [19]. The registration of PE in MBRN was found to have an average positive predictive value of $88 \%$, ranging from 80 to above $90 \%$ during these years.

The exposure information (PE) was collected prior to eventual dispensing of antihypertensive drugs. Therefore, selection and recall bias were eliminated. The outcome of the study (dispensing of antihypertensive drugs) was based on NorPD using reimbursed prescriptions (71\% of the first dispensed drugs among those included were reimbursed).

Date of dispensing medications was known, but we did not know whether the dispensed drugs were actually used, or exactly when they were used. Further, we were not able to capture medications received by women during a possible hospital stay. However, women given antihypertensive drugs during hospitalization will usually be issued a prescription when leaving the hospital for continued use at least for some time. All these prescriptions will be captured when dispensed, also at hospital pharmacies. Unlike some European countries, also prescriptions issued in private health care were captured. 
Table 2 Observed person-time, in the Norwegian Prescription Database, according to characteristic of the previous births ${ }^{\mathrm{a}}$

\begin{tabular}{|c|c|c|c|c|}
\hline & $\begin{array}{l}\text { Number of women who } \\
\text { were dispensed antihypertensive } \\
\text { drugs during follow-up }\end{array}$ & Person-years & $\%$ & $\begin{array}{l}\text { Rate of dispensed } \\
\text { antihypertensive drugs per } \\
1,000 \text { person-years }\end{array}$ \\
\hline \multicolumn{5}{|l|}{ Pregnancies } \\
\hline First pregnancy & 32,413 & $1,524,541$ & 23 & 21.3 \\
\hline Second pregnancy & 73,404 & $3,073,281$ & 47 & 23.9 \\
\hline Third pregnancy & 38,165 & $1,540,006$ & 23 & 24.8 \\
\hline Fourth pregnancy or more & 11,996 & 426,383 & 6 & 28.1 \\
\hline \multicolumn{5}{|l|}{ Preeclampsia } \\
\hline No preeclamptic pregnancies & 142,680 & $6,237,038$ & 95 & 22.9 \\
\hline Preeclampsia once & 11,636 & 295,603 & 5 & 39.4 \\
\hline Preeclampsia twice or more & 1662 & 31,570 & 0 & 52.6 \\
\hline \multicolumn{5}{|l|}{ Time since first birth (years) } \\
\hline $0-9$ & 14,660 & $1,699,935$ & 26 & 8.6 \\
\hline $10-19$ & 27,610 & $1,818,055$ & 28 & 15.2 \\
\hline $20-29$ & 41,526 & $1,521,496$ & 23 & 27.3 \\
\hline $30-39$ & 58,443 & $1,305,013$ & 20 & 44.8 \\
\hline $40-49$ & 13,739 & 219,714 & 3 & 62.5 \\
\hline Total & 155,978 & $6,564,212$ & 100 & 23.8 \\
\hline
\end{tabular}

${ }^{a}$ A woman may be included in more than one category due to multiple births

Table 3 Hazard ratios (HR) with $95 \%$ CI of being dispensed antihypertensive drugs, from time-dependent Cox regression analyses

\begin{tabular}{lllll}
\hline & HR & $95 \%$ CI & HR $^{\mathrm{a}}$ & $95 \%$ CI \\
\hline Year of birth (mother) & & & & \\
$<1950$ & 1.0 & Reference & 1.0 & Reference \\
$1950-1959$ & 0.8 & $0.8-0.8$ & 0.9 & $0.9-0.9$ \\
$1960-1969$ & 0.7 & $0.7-0.7$ & 0.9 & $0.9-0.9$ \\
$1970-1979$ & 0.6 & $0.6-0.6$ & 0.9 & $0.9-1.0$ \\
$1980-$ & 0.5 & $0.5-0.6$ & 0.9 & $0.9-1.0$ \\
Parity & & & & \\
No previous pregnancies & 1.0 & Reference & 1.0 & Reference \\
One previous pregnancy & 0.8 & $0.8-0.8$ & 0.8 & $0.8-0.8$ \\
Two previous pregnancies & 0.7 & $0.7-0.8$ & 0.8 & $0.8-0.8$ \\
Three previous pregnancies & 0.8 & $0.7-0.8$ & 0.8 & $0.8-0.8$ \\
Four or more previous pregnancies & 0.8 & $0.7-0.8$ & 0.8 & $0.8-0.8$ \\
Preeclampsia & & & & \\
No preeclamptic pregnancies & 1.0 & Reference & 1.0 & Reference \\
Preeclampsia once & 2.0 & $1.9-2.0$ & 2.0 & $2.0-2.0$ \\
Preeclampsia twice or more & 2.7 & $2.6-2.9$ & 2.8 & $2.7-3.0$ \\
\hline
\end{tabular}

${ }^{\text {a }}$ Adjusted for maternal age, age at first birth, year of childbirth and number of previous pregnancies with preeclampsia in addition to the variables presented in the table. Adjustments were made for each birth in each woman

b A woman may be included in more than one category due to multiple births
In this study, we used dispensed antihypertensive drugs as an indication of hypertension. To ascertain that the prescriptions were given to treat hypertension, we only used dispensed drugs that were reimbursed, indicating that the drugs were used for a chronic disease. Diabetes type II has been shown to be associated with obesity and use of antihypertensive drugs [20]. Adjustment for diabetes did only change the estimates moderately, but all types of diabetes (type I, type II, unspecified and gestational diabetes) were associated with later use of antihypertensive drugs. Another important risk factor for cardiovascular disease, smoking, reduces the risk of mild forms of PE [21]. Since 1999, maternal smoking habits have been registered in MBRN. However, adjusting for maternal smoking, or restricting the analyses to non-smokers only, did not change 
Table 4 Hazard ratios (HR) with $95 \%$ CI of being dispensed antihypertensive drugs after preterm and term preeclampsia in one or two or more pregnancies, mutually adjusted, from time-dependent Cox regression analyses

\begin{tabular}{lcllll}
\hline & \multicolumn{2}{l}{$1967-2012$} & & \multicolumn{2}{l}{$1999-2012$} \\
\cline { 2 - 3 } & $\mathrm{HR}^{\mathrm{a}}$ & $95 \% \mathrm{CI}$ & & $\mathrm{HR}^{\mathrm{a}}$ & $95 \% \mathrm{CI}$ \\
\hline Preterm preeclampsia & & & & \\
None & 1.0 & Reference & & 1.0 & Reference \\
One & 2.2 & $2.1-2.3$ & & 3.3 & $3.1-3.5$ \\
Two or more & 2.8 & $2.4-3.3-9.8$ & & 4.3 & $3.5-5.2$ \\
Term preeclampsia & & & & \\
None & 1.0 & Reference & & 1.0 & Reference \\
One & 1.9 & $1.9-1.9$ & & 2.5 & $2.4-2.6$ \\
Two or more & 2.6 & $2.5-2.8$ & & 3.4 & $3.1-3.8$ \\
\hline
\end{tabular}

${ }^{a}$ Adjusted for maternal age, age at first birth and year of childbirth

b Preterm preeclampsia: preeclampsia and pregnancy length $<37$ completed weeks

c Term preeclampsia: preeclampsia and pregnancy length $\geq 37$ completed weeks

the association between PE and later antihypertensive drug prescriptions, although maternal risk of being dispensed antihypertensive drugs was more than $25 \%$ higher in smoking mothers compared to non-smoking mothers in this period. These results indicate that maternal diabetes and smoking are not important when studying the relation between PE and later maternal hypertension.

We assumed that women not being dispensed antihypertensive drugs with reimbursement codes during the first 6 months of 2004, had not used these drugs previously. With this assumption, we lost women giving birth in the earlier years of MBRN who only had a transient need of antihypertensive drugs during the years before 2004 .

All births with registered maternal chronic hypertension or maternal heart disease before pregnancy were excluded. Other diseases may, however, predispose to PE as well. Sensitivity analyses also excluding rheumatoid arthritis and chronic renal disease were performed, but results were similar.

In studies based on data from large population-based registries, the presence of residual confounding cannot be ruled out, because the information recorded is often grouped in broad categories. However, adjustment for the variables included influenced the results only moderately. Hence, our results should be replicated in studies including more detailed information.

The risk of later maternal hypertension increased with the number of pregnancies with both preterm and term PE in our study. In both the review by Bellamy et al. [4] and by Brown et al. [11] the studies included had shorter follow-up. In our study, the HR of hypertension associated with PE decreased consistently by time since first birth, explaining the somewhat lower estimates in our study.

Magnussen et al. [17] concluded that women with hypertensive disorders during pregnancy were more likely to develop substantially higher blood pressure several years after pregnancy, and that the risk increased with the number of preeclamptic events. In our study, we found an increased risk of being dispensed antihypertensive drugs about 40 years after preeclamptic pregnancies. We also found increased risk with number of preeclamptic pregnancies. Magnussen et al. [17] did not find substantial difference in the cardiovascular risk factor profile between women who had experienced preterm and term PE. We, however, observed that women with preterm PE experienced a higher risk of being dispensed antihypertensive drugs than women with term PE, at least during the first 10 years after the pregnancy.

Romundstad et al. [18] have suggested that the association between hypertension in pregnancy and cardiovascular risk factors could to a large extent be attributed to risk factors present before pregnancy. This might be the case, but a history of PE may nevertheless serve as a screening tool for later health problems.
Table 5 Hazard ratios (HR) with $95 \%$ CI of being dispensed antihypertensive drugs after preeclamptic pregnancies in one or two or more pregnancies by time since first pregnancy, from timedependent Cox regression analyses

\begin{tabular}{llllll}
\hline $\begin{array}{l}\text { Years since } \\
\text { first pregnancy }\end{array}$ & \multicolumn{2}{l}{ One preeclamptic pregnancy $^{\mathrm{a}}$} & & \multicolumn{2}{l}{ Two or more preeclamptic pregnancies $^{\mathrm{a}}$} \\
\cline { 2 - 3 } & HR & $95 \% \mathrm{CI}$ & & HR & $95 \%$ CI \\
\hline $0-4$ & 5.7 & $5.4-6.1$ & & 10 & $8.1-12$ \\
$5-9$ & 2.5 & $2.4-2.7$ & & 4.5 & $3.9-5.2$ \\
$10-14$ & 2.3 & $2.2-2.4$ & & 3.6 & $3.2-4.1$ \\
$15-19$ & 2.1 & $2.0-2.2$ & & 3.0 & $2.7-3.4$ \\
$20-24$ & 1.9 & $1.9-2.1$ & & 2.7 & $2.4-3.1$ \\
$25-29$ & 1.8 & $1.7-1.9$ & & 2.5 & $2.2-2.8$ \\
$30-34$ & 1.6 & $1.5-1.7$ & & 2.1 & $1.8-2.5$ \\
$35-39$ & 1.4 & $1.4-1.5$ & & 1.9 & $1.6-2.2$ \\
$40-44$ & 1.3 & $1.2-1.4$ & & 1.6 & $1.1-2.1$ \\
\hline
\end{tabular}

${ }^{a}$ Additionally adjusted for maternal age, age at first birth and year of childbirth 
In conclusion, the HR of later use of antihypertensive drugs increased with the number of preeclamptic pregnancies, and in the first 10 years the HR was higher after a preterm than a term preeclamptic pregnancy. The excess risk decreased with time since first birth, but was still increased after 40 years.

Conflict of interest The authors declare that they have no conflicts of interest.

Open Access This article is distributed under the terms of the Creative Commons Attribution License which permits any use, distribution, and reproduction in any medium, provided the original author(s) and the source are credited.

\section{References}

1. The Medical Birth Registry of Norway Statistics. 2013.

2. Drost JT, Maas AH, van Eyck J, et al. Preeclampsia as a femalespecific risk factor for chronic hypertension. Maturitas. 2010;67:321-6.

3. Edlow AG, Srinivas SK, Elovitz MA. Investigating the risk of hypertension shortly after pregnancies complicated by preeclampsia. Am J Obstet Gynecol. 2009;200:e60-2.

4. Bellamy L, Casas JP, Hingorani AD, et al. Pre-eclampsia and risk of cardiovascular disease and cancer in later life: systematic review and meta-analysis. BMJ. 2007;335:974.

5. Tranquilli AL, Landi B, Giannubilo SR, et al. Preeclampsia: no longer solely a pregnancy disease. Pregnancy Hypertens Int $\mathbf{J}$ Women's Cardiovasc Health. 2012;2:350-7.

6. Skjærven R, Wilcox AJ, Klungsøyr K, et al. Cardiovascular mortality after pre-eclampsia in one child mothers: prospective, population based cohort study. BMJ. 2012;345:e7677.

7. Chen CW, Jaffe IZ, Karumanchi SA. Pre-eclampsia and cardiovascular disease. Cardiovasc Res. 2014;101:579-86.
8. Mutter WP, Karumanchi SA. Molecular mechanisms of preeclampsia. Microvasc Res. 2008;75:1-8.

9. Williams D. Pregnancy: a stress test for life. Curr Opin Obstet Gynecol. 2003;15:465-71.

10. Drost JT, van der Schouw YT, Maas AH, et al. Longitudinal analysis of cardiovascular risk parameters in women with a history of hypertensive pregnancy disorders: the Doetinchem Cohort Study. BJOG. 2013;120:1333-9.

11. Brown MC, Best KE, Pearce MS, et al. Cardiovascular disease risk in women with pre-eclampsia: systematic review and metaanalysis. Eur J Epidemiol. 2013;28:1-19.

12. Hammer $\mathrm{H}$. The central population registry in medical research. Tidsskr Nor Laegeforen. 2002;122:2550.

13. Furu K. Establishment of the nationwide Norwegian Prescription Database (NorPD) - new opportunities for research in pharmacoepidemiology in Norway. Nor J Epidemiol. 2008;18:129-36.

14. WHO Collaborating Centre for Drug Statistics Methodology. 2010.

15. Irgens LM. The Medical Birth Registry of Norway. Epidemiological research and surveillance throughout 30 years. Acta Obstet Gynecol Scand. 2000;79:435-9.

16. Skjærven R, Gjessing HK, Bakketeig LS. Birthweight by gestational age in Norway. Acta Obstet Gynecol Scand. 2000;79: 440-9.

17. Magnussen EB, Vatten LJ, Smith GD, et al. Hypertensive disorders in pregnancy and subsequently measured cardiovascular risk factors. Obstet Gynecol. 2009;114:961-70.

18. Romundstad PR, Magnussen EB, Smith GD, et al. Hypertension in pregnancy and later cardiovascular risk: common antecedents? Circulation. 2010;122:579-84.

19. Thomsen LC, Klungsøyr K, Roten LT, et al. Validity of the diagnosis of pre-eclampsia in the Medical Birth Registry of Norway. Acta Obstet Gynecol Scand. 2013;92:943-50.

20. Demaria AN. The multiple challenges of obesity. J Am Coll Cardiol. 2013;61:784-6.

21. Karumanchi SA, Levine RJ. How does smoking reduce the risk of preeclampsia? Hypertension. 2010;55:1100-1. 